\title{
Strange form factors of the nucleon with a nonlocal chiral effective Lagrangian
}

\author{
Fangcheng $\mathrm{He}^{1,2}$ and P. Wang ${ }^{1,3}$ \\ ${ }^{1}$ Institute of High Energy Physics, CAS, P. O. Box 918(4), Beijing 100049, China \\ ${ }^{2}$ University of Chinese Academy of Sciences, Beijing 100049, China \\ ${ }^{3}$ Theoretical Physics Center for Science Facilities, CAS, Beijing 100049, China
}

(Received 29 May 2018; published 9 August 2018)

\begin{abstract}
The strange form factors of the nucleon are studied with the nonlocal chiral effective Lagrangian. One loop contributions from both octet and decuplet intermediate states are included. The relativistic regulator is obtained by the nonlocal Lagrangian where the gauge link is introduced to guarantee the local gauge symmetry. With the kaon loop, the calculated charge form factor is positive, while the magnetic form factor is negative. The strange magnetic moment is $-0.041_{-0.014}^{+0.012}$ with $\Lambda=0.9 \pm 0.1$ determined from the nucleon electromagnetic form factors. Our results are comparable with the recent lattice simulation.
\end{abstract}

DOI: 10.1103/PhysRevD.98.036007

\section{INTRODUCTION}

It is well known that a complete characterization of nucleon substructure must go beyond three valence quarks. One of the great challenges of modern hadron physics is to unravel the precise role of hidden flavors in the structure of the nucleon. Strange quark contribution to the nucleon form factors has attracted a lot of interest because it is purely from the sea quark. The role of the sea remains a central issue in QCD, especially with respect to lattice QCD. There such terms involve so-called disconnected graphs, i.e., quark loops are connected only by gluons to the valence quarks.

Parity-violating electron scattering (PVES) has proven to be a valuable tool for experimentally determining the strange quark contribution to the electromagnetic form factors of the proton. Under the assumption of charge symmetry, one can deduce the strange electric or magnetic form factor $G_{E, M}^{s}\left(Q^{2}\right)$ from measurements of the corresponding proton and neutron electromagnetic form factors and the neutral-weak vector form factor of the proton, through its contribution to PVES. While PVES measurements are very challenging, a number of groups have succeeded, starting with SAMPLE at Bates [1] and then A4 at Mainz [2,3] and G0 [4] and HAPPEX [5-7] at Jefferson Lab. Up to now, the experiments have not provided an unambiguous confirmed answer to the sign of the strange

Published by the American Physical Society under the terms of the Creative Commons Attribution 4.0 International license. Further distribution of this work must maintain attribution to the author(s) and the published article's title, journal citation, and DOI. Funded by SCOAP ${ }^{3}$. form factors, although global analyses do tend to suggest that $G_{s}^{M}(0)<0$ is favored [8,9].

Theoretically, though QCD is the fundamental theory to describe strong interactions, it is difficult to study hadron physics using QCD directly. There are many phenomenological models, such as the cloudy bag model [10], the constituent quark model [11,12], the $1 / \mathrm{Nc}$ expansion approach [13], the perturbative chiral quark model $[14,15]$, the extended vector meson dominance model [16], the SU(3) chiral quark model [17], the quark-diquark model $[18,19]$, etc. Besides the above phenomenological models, heavy baryon and relativistic chiral perturbation theory have been widely applied to study the hadron spectrum and structure. Different regularization methods, such as the dimensional regularization, infrared regularization, extend on-mass-shell scheme (EOMS), etc, were used in the loop calculation. Especially, many recent calculations have been performed in the frame work of EOMS [20,21]. Though ChPT is a successful and systematic approach, for the nucleon electromagnetic form factors, it is only valid for $Q^{2}<0.1 \mathrm{GeV}^{2}$ [22]. When vector mesons are included, the result is close to the experiments with $Q^{2}$ less than $0.4 \mathrm{GeV}^{2}[23,24]$.

An alternative regularization method, namely finiterange-regularization (FRR) has been proposed. Inspired by quark models that account for the finite-size of the nucleon as the source of the pion cloud, effective field theory with FRR has been widely applied to extrapolate the vector meson mass, magnetic moments, magnetic form factors, strange form factors, charge radii, first moments of GPDs, nucleon spin, etc [25-40]. In the finite-rangeregularization, there is no cut for the energy integral. The regulator is not covariant and is in three-dimensional 
momentum space. This nonrelativistic regulator can only be applied with the heavy baryon ChPT.

We proposed a relativistic version for the finite-rangeregularization which makes it possible to study the hadron properties with relativistic chiral effective Lagrangian at large $Q^{2}$ [41,42]. The covariant regulator was generated from the nonlocal gauge invariant Lagrangian. As a result, the renormalized charge of proton (neutron) is 1 (0) with the additional diagrams obtained by the expansion of the gauge link. The nonlocal interaction generates both the regulator which makes the loop integral convergent and the $Q^{2}$ dependence of form factors at tree level. The obtained electric and magnetic form factors of nucleon are very close to the experimental data [42]. This was the first time to calculate the form factors precisely at relatively large $Q^{2}$ with chiral effective Lagrangian.

In this paper, we will apply the nonlocal chiral effective Lagrangian to study the strange form factors. The paper is organized in the following way. In Sec. II, we briefly introduce the chiral effective Lagrangian. The strange form factors are derived in Sec. III. Numerical results are presented in Sec. IV and finally, Sec. V is a summary.

\section{CHIRAL EFFECTIVE LAGRANGIAN}

The lowest order chiral Lagrangian for baryons, pseudoscalar mesons and their interaction can be written as $[43,44]$.

$$
\begin{aligned}
\mathcal{L}= & i \operatorname{Tr} \bar{B} \gamma_{\mu} \not D B-m_{B} \operatorname{Tr} \bar{B} B+\bar{T}_{\mu}^{a b c}\left(i \gamma^{\mu \nu \alpha} D_{\alpha}-m_{T} \gamma^{\mu \nu}\right) T_{\nu}^{a b c} \\
& +\frac{f^{2}}{4} \operatorname{Tr} \partial_{\mu} \Sigma \partial^{\mu} \Sigma^{+}+D \operatorname{Tr} \bar{B} \gamma_{\mu} \gamma_{5}\left\{A_{\mu}, B\right\} \\
& +F \operatorname{Tr} \bar{B} \gamma_{\mu} \gamma_{5}\left[A_{\mu}, B\right]+\left[\frac{\mathcal{C}}{f} \epsilon^{a b c} \bar{T}_{\mu}^{a d e}\left(g^{\mu \nu}+z \gamma_{\mu} \gamma_{\nu}\right) B_{c}^{e} \partial_{\nu} \phi_{b}^{d}\right. \\
& + \text { H.C. }]+H \bar{T}^{\mu} \gamma_{\nu} \gamma^{5} A^{\nu} T_{\mu},
\end{aligned}
$$

where $D, F, \mathcal{C}$, and $H$ are the coupling constants. The chiral covariant derivative $\mathcal{D}_{\mu}$ is defined as $\mathcal{D}_{\mu} B=\partial_{\mu} B+\left[V_{\mu}, B\right]$. The pseudoscalar meson octet couples to the baryon field through the vector and axial vector combinations as

$V_{\mu}=\frac{1}{2}\left(\zeta \partial_{\mu} \zeta^{\dagger}+\zeta^{\dagger} \partial_{\mu} \zeta\right)+\frac{1}{2} i e \mathcal{A}_{\mu}\left(\zeta^{\dagger} Q \zeta+\zeta Q \zeta^{\dagger}\right)$,

$A_{\mu}=\frac{1}{2}\left(\zeta \partial_{\mu} \zeta^{\dagger}-\zeta^{\dagger} \partial_{\mu} \zeta\right)-\frac{1}{2} e \mathcal{A}_{\mu}\left(\zeta Q \zeta^{\dagger}-\zeta^{\dagger} Q \zeta\right)$,

where the matrix $Q$ is defined as $Q=\operatorname{diag}\{2 / 3,-1 / 3$, $-1 / 3\}$ and

$$
\Sigma=\zeta^{2}=e^{2 i \phi / f}, \quad f=93 \mathrm{MeV} .
$$

The matrix of pseudoscalar fields $\phi$ is expressed as $\phi=\frac{1}{\sqrt{2}}\left(\begin{array}{lcr}\frac{1}{\sqrt{2}} \pi^{0}+\frac{1}{\sqrt{6}} \eta & \pi^{+} & K^{+} \\ \pi^{-} & -\frac{1}{\sqrt{2}} \pi^{0}+\frac{1}{\sqrt{6}} \eta & K^{0} \\ K^{-} & \bar{K}^{0} & -\frac{2}{\sqrt{6}} \eta\end{array}\right)$.

$\mathcal{A}_{\mu}$ is the photon field. The covariant derivative $D_{\mu}$ in the decuplet part is defined as $D_{\nu} T_{\mu}^{a b c}=\partial_{\nu} T_{\mu}^{a b c}+\left(\Gamma_{\nu}, T_{\mu}\right)^{a b c}$, where $\Gamma_{\nu}$ is the chiral connection [45] defined as $\left(X, T_{\mu}\right)=(X)_{d}^{a} T_{\mu}^{d b c}+(X)_{d}^{b} T_{\mu}^{a d c}+(X)_{d}^{c} T_{\mu}^{a b d} \cdot \gamma^{\mu \nu \alpha}, \gamma^{\mu \nu}$ are the antisymmetric matrices expressed as

$$
\gamma^{\mu \nu}=\frac{1}{2}\left[\gamma^{\mu}, \gamma^{\nu}\right] \quad \text { and } \quad \gamma^{\mu \nu \rho}=\frac{1}{4}\left\{\left[\gamma^{\mu}, \gamma^{\nu}\right], \gamma^{\rho}\right\}
$$

In the chiral $S U(3)$ limit, the octet and decuplet baryons will have the same mass $m_{B}$ and $m_{T}$. In our calculation, we use the physical masses for baryon octets and decuplets. The explicit form of the baryon octet is written as

$$
B=\left(\begin{array}{lcr}
\frac{1}{\sqrt{2}} \Sigma^{0}+\frac{1}{\sqrt{6}} \Lambda & \Sigma^{+} & p \\
\Sigma^{-} & -\frac{1}{\sqrt{2}} \Sigma^{0}+\frac{1}{\sqrt{6}} \Lambda & n \\
\Xi^{-} & \Xi^{0} & -\frac{2}{\sqrt{6}} \Lambda
\end{array}\right) .
$$

For the baryon decuplets, there are three indices, defined as

$T_{111}=\Delta^{++}, \quad T_{112}=\frac{1}{\sqrt{3}} \Delta^{+}, \quad T_{122}=\frac{1}{\sqrt{3}} \Delta^{0}$,

$T_{222}=\Delta^{-}, \quad T_{113}=\frac{1}{\sqrt{3}} \Sigma^{*,+}, \quad T_{123}=\frac{1}{\sqrt{6}} \Sigma^{*, 0}$,

$T_{223}=\frac{1}{\sqrt{3}} \Sigma^{*,-}, \quad T_{133}=\frac{1}{\sqrt{3}} \Xi^{*, 0}$,

$T_{233}=\frac{1}{\sqrt{3}} \Xi^{*,-}, \quad T_{333}=\Omega^{-}$.

We should mention that permutations of the indices give rise to the same physical fields. For example, $T_{112}=$ $T_{121}=T_{211}=\Delta^{+} / \sqrt{3}$. The octet, decuplet, and octetdecuplet transition magnetic moment operators are needed in the one loop calculation of nucleon electromagnetic form factors. The baryon octet anomalous magnetic Lagrangian is written as

$$
\mathcal{L}=\frac{e}{4 m_{N}}\left(c_{1} \operatorname{Tr} \bar{B} \sigma^{\mu \nu}\left\{F_{\mu \nu}^{+}, B\right\}+c_{2} \operatorname{Tr} \bar{B} \sigma^{\mu \nu}\left[F_{\mu \nu}^{+}, B\right]\right),
$$

where

$$
F_{\mu \nu}^{+}=-\frac{1}{2}\left(\zeta^{\dagger} F_{\mu \nu} Q \zeta+\zeta F_{\mu \nu} Q \zeta^{\dagger}\right) .
$$

At the lowest order, the Lagrangian will generate the following nucleon anomalous magnetic moments:

$$
F_{2}^{p}=\frac{1}{3} c_{1}+c_{2}, \quad F_{2}^{n}=-\frac{2}{3} c_{1} .
$$


The transition magnetic operator is

$$
\mathcal{L}=i \frac{e}{4 m_{N}} \mu_{T} F_{\mu \nu}\left(\epsilon_{i j k} Q_{j}^{i} \bar{B}_{m}^{j} \gamma^{\mu} \gamma_{5} T^{\nu, k l m}+\epsilon^{i j k} Q_{i}^{l} \bar{T}_{k l m}^{\mu} \gamma^{\nu} \gamma_{5} B_{j}^{m}\right)
$$

The effective decuplet anomalous magnetic moment operator can be expressed as effective Lagrangian

$$
\mathcal{L}=\frac{e F_{2}^{T}}{4 m_{T}} \bar{T}_{\mu}^{a b c} \sigma^{\rho \sigma} F_{\rho \sigma} T_{\mu}^{a b c} .
$$

In the quark model, the baryon magnetic moments can also be written in terms of quark magnetic moments. For example, $\mu_{p}=\frac{4}{3} \mu_{u}-\frac{1}{3} \mu_{d}, \mu_{n}=\frac{4}{3} \mu_{d}-\frac{1}{3} \mu_{u}, \mu_{\Delta^{++}}=3 \mu_{u}$. Using $\mu_{u}=-2 \mu_{d}=-2 \mu_{s}, \mu_{T}, F_{2}^{T}$ and $\mu_{q}$ can be written in terms of $c_{1}$. For example,

$$
\mu_{T}=4 c_{1}, \quad F_{2}^{\Delta^{++}}=2 c_{1}-2, \quad \mu_{s}=-\frac{1}{3} c_{1} .
$$

The strange quark contribution to the hyperons at tree level can be written as [46]

$$
\mu_{\Sigma^{+}}^{s}=\mu_{\Sigma^{-}}^{s}=\mu_{\Sigma^{0}}^{s}=\mu_{s}, \quad \mu_{\Lambda}^{s}=-3 \mu_{s} .
$$

Similarly, the strange quark contribution to the decuplet and transition magnetic moments at tree level can be written as [47]

$$
\begin{gathered}
\mu_{\Sigma^{*,+}}^{s}=\mu_{\Sigma^{*,-}}^{s}=\mu_{\Sigma^{*, 0}}^{s}=-3 \mu_{s}, \\
\mu_{\Sigma^{*,+} \Sigma^{+}}^{s}=-\mu_{\Sigma^{*,-} \Sigma^{-}}^{s}=-\mu_{\Sigma^{*, 0} \Sigma^{0}}^{s}=-2 \sqrt{2} \mu_{s},
\end{gathered}
$$

Therefore, all the strange quark contribution to the magnetic moment at tree level is associated with the parameter $\mu_{s}$. With the $S U(6)$ symmetry in quark model it can be related to $c_{1}$ as in Eq. (13). Following the usual convention, the charge of the strange quark is taken to be 1 . It is the $U(1)$ charge associated with the gauging of strangeness. Now we construct the nonlocal Lagrangian which will generate the covariant regulator. The gauge invariant nonlocal Lagrangian can be obtained using the method in $[41,42,48]$. For instance, the local interaction including kaon can be written as

$$
\begin{aligned}
S_{K}^{\text {local }}= & -\int d^{4} x \frac{D+3 F}{\sqrt{12} f} \bar{p}(x) \gamma^{\mu} \gamma_{5} \Lambda(x) \\
& \times\left(\partial_{\mu}+i e \mathcal{A}_{\mu}^{s}(x)\right) K^{+}(x),
\end{aligned}
$$

where $\mathcal{A}_{\mu}^{s}(x)$ is the external field interacting the strange quark. The nonlocal action for this interaction is expressed as

$$
\begin{aligned}
S_{K}^{n l}= & -\int d^{4} x \int d^{4} y \frac{D+F}{\sqrt{12} f} \bar{p}(x) \gamma^{\mu} \gamma_{5} \Lambda(x)\left(\partial_{\mu}+i e \int d^{4} a \mathcal{A}_{\mu}^{s}(x-a) F(a)\right) \\
& \times \exp \left[i e \int_{x}^{y} d z^{\nu} \int d^{4} a \mathcal{A}_{\nu}^{s}(z-a) F(a)\right] K^{+}(y) F(x-y),
\end{aligned}
$$

where $F(x-y)$ is the correlation function between nucleon and meson field. For point particle, $F(x-y)$ is a $\delta$ function. Because nucleon is a nonpoint object, we introduce the correlation function which describes the connection between the meson or photon and nucleon in different coordinates. The nonlocal behavior is mainly due to the size effect of the nucleon. Therefore, for nucleon-meson and nucleon-photon interactions, the same correlation function is chosen. To guarantee the gauge invariant, the gauge link is introduced in the above Lagrangian. The regulator can be generated automatically with correlation function. With the same idea, the nonlocal interaction between baryons and $\mathcal{A}_{\mu}^{s}(x)$ can also be obtained. For example, the local interaction between $\Lambda$ and external field is written as

$$
\begin{aligned}
\mathcal{L}_{\Lambda}^{\text {local }}= & -e \bar{\Lambda}(x) \gamma^{\mu} \Lambda(x) \mathcal{A}_{\mu}^{s}(x) \\
& +\frac{\left(c_{1}-1\right) e}{4 m_{\Lambda}} \bar{\Lambda}(x) \sigma^{\mu \nu} \Lambda(x) F_{\mu \nu}^{s}(x) .
\end{aligned}
$$

The corresponding nonlocal Lagrangian is expressed as

$$
\begin{aligned}
\mathcal{L}_{\Lambda}^{n l}= & -e \int d^{4} a \bar{\Lambda}(x) \gamma^{\mu} \Lambda(x) \mathcal{A}_{\mu}^{s}(x-a) F_{1}(a) \\
& +\frac{\left(c_{1}-1\right) e}{4 m_{\Lambda}} \int d^{4} a \bar{\Lambda}(x) \sigma^{\mu \nu} \Lambda(x) F_{\mu \nu}^{s}(x-a) F_{2}(a),
\end{aligned}
$$

where $F_{1}(a)$ and $F_{2}(a)$ is the correlation function for the nonlocal strange electric and magnetic interactions. The form factors at tree level which are momentum dependent can be easily obtained with the Fourier transformation of the correlation function. The simplest choice is to assume that the correlation function of the strange electromagnetic vertex is the same as that of the lambda-kaon vertex, i.e., $F_{1}(a)=F_{2}(a)=F(a)$. Therefore, the Dirac and Pauli form factors will have the same dependence on the momentum transfer at tree level. The better choice is to assume that the charge and magnetic form factors at tree level have the same the momentum dependence as 
lambda-kaon vertex, i.e., $G_{M}^{\text {tree }}(p)=c_{1} G_{E}^{\text {tree }}(p)=c_{1} \tilde{F}(p)$, where $\tilde{F}(p)$ is the Fourier transformation of the correlation function $F(a)$ [42]. The corresponding function of $\tilde{F}_{1}(q)$ and $\tilde{F}_{2}(q)$ is then expressed as

$$
\begin{aligned}
& \tilde{F}_{1}^{p}(q)=\tilde{F}(q) \frac{4 m_{\Lambda}^{2}+c_{1} Q^{2}}{4 m_{\Lambda}^{2}+Q^{2}}, \\
& \tilde{F}_{2}^{p}(q)=\tilde{F}(q) \frac{4 m_{\Lambda}^{2}}{4 m_{\Lambda}^{2}+Q^{2}},
\end{aligned}
$$

From the above equations, one can see that in the heavy baryon limit, these two choices are equivalent. The nonlocal Lagrangian is invariant under the following gauge transformation

$$
\begin{aligned}
K^{+}(y) & \rightarrow e^{-i \alpha(y)} K^{+}(y), \\
\Lambda(x) & \rightarrow e^{i \alpha(x)} \Lambda(x), \\
\mathcal{A}_{\mu}^{s}(x) & \rightarrow \mathcal{A}_{\mu}^{s}(x)+\frac{1}{e} \partial_{\mu} \alpha^{\prime}(x),
\end{aligned}
$$

where $\alpha(x)=\int d a \alpha^{\prime}(x-a) F(a)$. From Eq. (18), two kinds of couplings between hadrons and external field can be obtained. One is the normal one expressed as

$$
\begin{aligned}
S^{\text {nor }}= & -i e \int d^{4} x \int d^{4} y \frac{D+F}{\sqrt{12} f} \bar{p}(x) \gamma^{\mu} \gamma_{5} \Lambda(x) \\
& \times F(x-y) K^{+}(y) \int d^{4} a \mathcal{A}_{\mu}^{s}(x-a) F(a) .
\end{aligned}
$$

This interaction is similar as the traditional local Lagrangian except the correlation function. The other one is the additional interaction obtained by the expansion of the gauge link, expressed as

$$
\begin{aligned}
S^{\mathrm{add}}= & -i e \int d^{4} x \int d^{4} y \frac{D+F}{\sqrt{12} f} \bar{p}(x) \gamma^{\mu} \gamma_{5} \Lambda(x) \\
& \times \partial_{\mu}\left(F(x-y) \int_{x}^{y} d z^{\nu} \int d^{4} a \mathcal{A}_{\nu}^{s}(z) F(z-a) K^{+}(y)\right) .
\end{aligned}
$$

The additional interaction is important guarantee the local gauge symmetry resulting the net strangeness of nucleon zero.

\section{STRANGE FORM FACTORS}

The strange quark contribution to the Dirac and Pauli form factors are defined as

$$
\begin{aligned}
& \left\langle N\left(p^{\prime}\right)\left|J_{\mu}^{s}\right| N(p)\right\rangle \\
& \quad=\bar{u}\left(p^{\prime}\right)\left\{\gamma^{\mu} F_{1}^{s}\left(Q^{2}\right)+\frac{i \sigma^{\mu \nu} q_{\nu}}{2 m_{N}} F_{2}^{s}\left(Q^{2}\right)\right\} u(p),
\end{aligned}
$$

where $q=p^{\prime}-p$ and $Q^{2}=-q^{2}$. The combination of the above form factors can generate electric and magnetic form factors contributed from strange quark

$$
\begin{aligned}
& G_{E}^{s}\left(Q^{2}\right)=F_{1}^{s}\left(Q^{2}\right)-\frac{Q^{2}}{4 m_{N}^{2}} F_{2}^{s}\left(Q^{2}\right), \\
& G_{M}^{s}\left(Q^{2}\right)=F_{1}^{s}\left(Q^{2}\right)+F_{2}^{s}\left(Q^{2}\right) .
\end{aligned}
$$

According to the Lagrangian, the one loop Feynman diagrams which contribute to the strange form factors are plotted in Fig 1.

In this section, we will only show the expressions for the intermediate octet baryon part. For the intermediate decuplet baryon part, the expressions are written in the Appendix. In Fig. 1(a), the external field couples to the meson. The contribution of Fig. 1(a) to the matrix element in Eq. (25) is expressed as

$$
\Gamma_{a}^{\mu(p)}=\frac{(3 F+D)^{2}}{12 f^{2}} I_{a K}^{\Lambda}+\frac{3(D-F)^{2}}{4 f^{2}} I_{a K}^{\Sigma},
$$

where the integral $I_{a K}^{\Lambda}$ is expressed as

$$
\begin{aligned}
I_{a K}^{\Lambda}= & \bar{u}\left(p^{\prime}\right) \tilde{F}(q) \int \frac{d^{4} k}{(2 \pi)^{4}}(\not k+\not q) \gamma_{5} \tilde{F}(q+k) \\
& \times \frac{1}{D_{K}(k+q)}(2 k+q)^{\mu} \frac{1}{D_{K}(k)} \frac{1}{\not p-\not k-m_{\Lambda}} \\
& \times\left(-\not k \gamma_{5}\right) \tilde{F}(k) u(p)
\end{aligned}
$$

and $D_{K}(k)$ is defined as

$$
D_{K}(k)=k^{2}-M_{K}^{2}+i \epsilon .
$$

$m_{\Lambda}$ and $M_{K}$ are the masses for the intermediate $\Lambda$ hyperon and $K$ meson, respectively. The integral $I_{a K}^{\Sigma}$ is the same as $I_{a K}^{\Lambda}$ except the intermediate hyperon mass $m_{\Lambda}$ is replaced by $m_{\Sigma}$. Therefore, here we only show the expressions for $\Lambda$ hyperon. In Fig. 1(b), the external field couples to the intermediate hyperons with electric interaction. The contribution of this diagram is expressed as

$$
\begin{aligned}
\Gamma_{b}^{\mu(p)}= & \frac{(3 F+D)^{2}}{12 f^{2}} \frac{4 m_{\Lambda}^{2}+c_{1} Q^{2}}{4 m_{\Lambda}^{2}+Q^{2}} I_{b K}^{\Lambda} \\
& +\frac{3(D-F)^{2}}{4 f^{2}} \frac{12 m_{\Sigma}^{2}-c_{1} Q^{2}}{12 m_{\Sigma}^{2}+3 Q^{2}} I_{b K}^{\Sigma},
\end{aligned}
$$

where the integral $I_{b K}^{\Lambda}$ is written as 


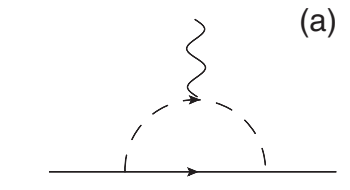

(c)

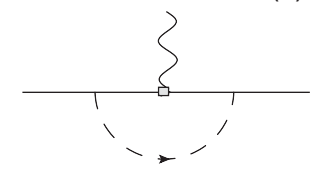

(e)

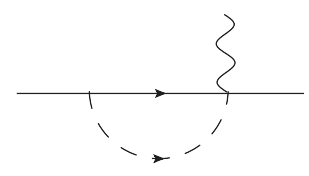

(g)

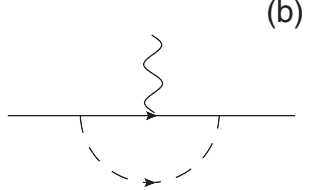

(d)

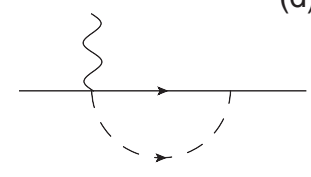

(f)

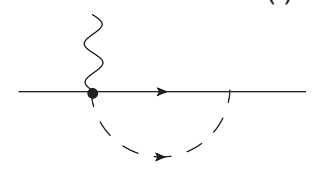

(n)

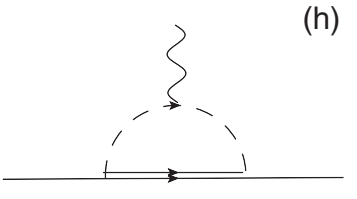

(h)

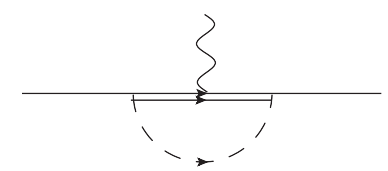

(j)

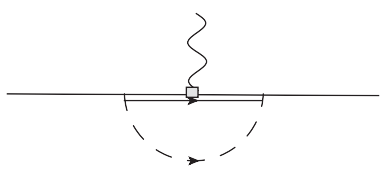

(I)

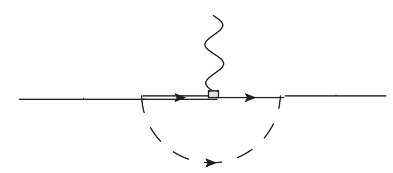

(m)

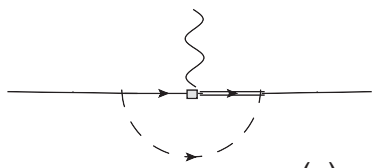

(o)

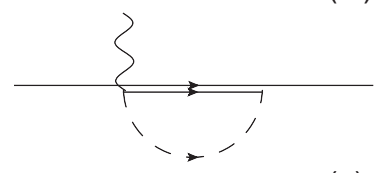

(p)
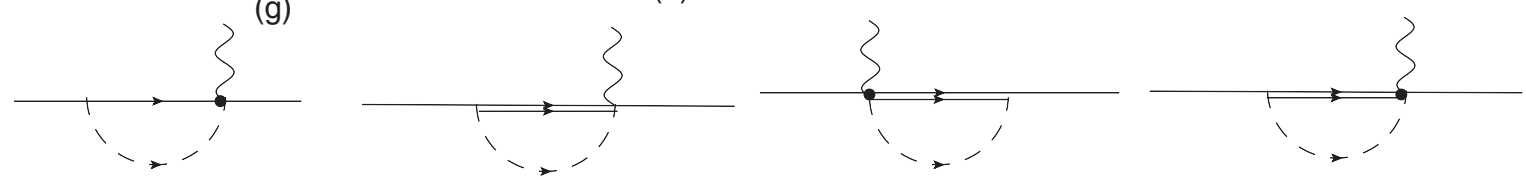

FIG. 1. One-loop contributions to the proton strange electromagnetic form factor. The solid, double-solid, dashed, and wavy lines are for the octet baryons, decuplet baryons, pseudoscalar mesons, and photons, respectively. The rectangle and blackdot represent magnetic and additional interacting vertex.

$$
\begin{aligned}
I_{b K}^{\Lambda}= & \bar{u}\left(p^{\prime}\right) \tilde{F}(q) \int \frac{d^{4} k}{(2 \pi)^{4}} \not k \gamma_{5} \tilde{F}(k) \frac{1}{D_{K}(k)} \frac{1}{\not p^{\prime}-\not k-m_{\Lambda}} \\
& \times \gamma^{\mu} \frac{1}{\not p-\not k-m_{\Lambda}} \frac{\not k \gamma_{5}}{\sqrt{2} f} \tilde{F}(k) u(p) .
\end{aligned}
$$

Figure 1(c) is similar as Fig. 1(b) except for the magnetic interaction. The contribution of this diagram is written as

$$
\begin{aligned}
\Gamma_{c}^{\mu(p)}= & \frac{(3 F+D)^{2}}{12 f^{2}} \frac{4\left(c_{1}-1\right) m_{\Lambda}^{2}}{4 m_{\Lambda}^{2}+Q^{2}} I_{c K}^{\Lambda} \\
& -\frac{3(D-F)^{2}}{4 f^{2}} \frac{\left(4 c_{1}+12\right) m_{\Sigma}^{2}}{12 m_{p}^{2}+3 Q^{2}} I_{c K}^{\Sigma},
\end{aligned}
$$

where $I_{c K}^{\Lambda}$ is expressed as

$$
\begin{aligned}
I_{c K}^{\Lambda}= & \bar{u}\left(p^{\prime}\right) \tilde{F}(q) \int \frac{d^{4} k}{(2 \pi)^{4}} \not k \gamma_{5} \tilde{F}(k) \frac{1}{\not p^{\prime}-\not k-m_{\Lambda}} \frac{\sigma^{\mu \nu} q_{\nu}}{2 m_{\Lambda}} \\
& \times \frac{1}{\not \supset-\not k-m_{\Lambda}} \frac{i}{D_{K}(k)} \not k \gamma_{5} \tilde{F}(k) u(p) .
\end{aligned}
$$

Figures 1(d) and 1(e) are the Kroll-Ruderman diagrams. The contribution from these two diagrams is written as

$$
\Gamma_{d+e}^{\mu(p)}=\frac{(3 F+D)^{2}}{12 f^{2}} I_{(d+e) K}^{\Lambda}+\frac{3(D-F)^{2}}{4 f^{2}} I_{(d+e) K}^{\Sigma},
$$

where

$$
\begin{aligned}
I_{(d+e) K}^{\Lambda}= & \bar{u}\left(p^{\prime}\right) \tilde{F}(q) \int \frac{d^{4} k}{(2 \pi)^{4}} \not k \gamma_{5} \tilde{F}(k) \frac{1}{\not p^{\prime}-\not k-m} \frac{1}{D_{K}(k)} \gamma^{\mu} \gamma_{5} \tilde{F}(k) u(p) \\
& +\bar{u}\left(p^{\prime}\right) \tilde{F}(q) \int \frac{d^{4} k}{(2 \pi)^{4}} \gamma^{\mu} \gamma_{5} \tilde{F}(k) \frac{1}{\not p-\not k-m} \frac{1}{D_{K}(k)} \not k \gamma_{5} \tilde{F}(k) u(p) .
\end{aligned}
$$

These two diagrams only have contribution in the relativistic cases. In the heavy baryon limit, they have no contribution to either electric or magnetic form factors. Figures 1(f) and 1(g) are the additional diagrams which generated from the expansion of the gauge link. The contribution of these two additional diagrams are expressed as

$$
\Gamma_{f+g}^{\mu(p)}=\frac{(3 F+D)^{2}}{12 f^{2}} I_{(f+g) K}^{\Lambda}+\frac{3(D-F)^{2}}{4 f^{2}} I_{(f+g) K}^{\Sigma},
$$


where

$$
\begin{aligned}
I_{(f+g) K}^{\Lambda}= & \bar{u}\left(p^{\prime}\right) \tilde{F}(q) \int \frac{d^{4} k}{(2 \pi)^{4}} \not k \gamma_{5} \tilde{F}(k) \frac{1}{\not p^{\prime}-\not k-m_{\Lambda}} \frac{1}{D_{K}(k)}(-\not k+\not q) \gamma_{5} \frac{(2 k-q)^{\mu}}{2 k q-q^{2}}[\tilde{F}(k-q)-\tilde{F}(k)] u(p) \\
+ & \bar{u}\left(p^{\prime}\right) \tilde{F}(q) \int \frac{d^{4} k}{(2 \pi)^{4}}(\not k+\not h) \gamma_{5} \frac{(2 k+q)^{\mu}}{2 k q+q^{2}}[\tilde{F}(k+q)-\tilde{F}(k)] \frac{1}{\not p-\not k-m_{\Lambda}} \frac{1}{D_{K}(k)} \not k \gamma_{5} \tilde{F}(k) u(p) .
\end{aligned}
$$

Using FEYNCALC to simplify the $\gamma$ matrix algebra, we can get the separate expressions for the Dirac and Pauli form factors.

\section{NUMERICAL RESULTS}

In the numerical calculations, the parameters are chosen as $D=0.76$ and $F=0.5\left(g_{A}=D+F=1.26\right)$. The coupling constant $\mathcal{C}$ is chosen to be 1 which are the same as [49]. The off-shell parameter $z$ is chosen to be $z=-1$ [50]. The covariant regulator is chosen to be of a dipole form

$$
\tilde{F}(k)=\frac{\Lambda^{4}}{\left(k^{2}-M_{j}^{2}-\Lambda^{2}\right)^{2}},
$$

where $M_{j}$ is the mass of the corresponding meson and it is zero for photon. For example, for the nucleon-photon vertex, the function $\tilde{F}(q)$ is expressed as

$$
\tilde{F}(q)=\frac{\Lambda^{4}}{\left(q^{2}-\Lambda^{2}\right)^{2}} .
$$

For the nucleon-K-meson vertex, $\tilde{F}(k)$ is expressed as

$$
\tilde{F}(k)=\frac{\Lambda^{4}}{\left(k^{2}-M_{K}^{2}-\Lambda^{2}\right)^{2}} .
$$

Therefore, in this nonlocal Lagrangian, there are three parameters $c_{1}, c_{2}$, and $\Lambda$ to be determined. $\Lambda$ is chosen to

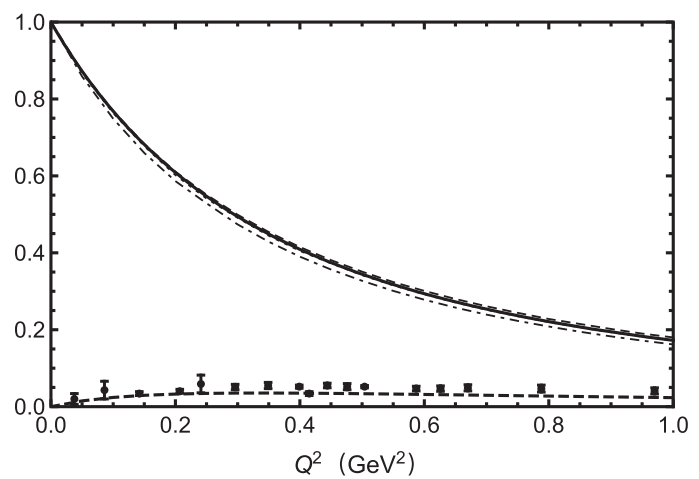

FIG. 2. Nucleon electromagnetic form factors versus momentum transfer $Q^{2}$. The solid line is for the empirical function $1 /\left(1+Q^{2} / 0.71 \mathrm{GeV}^{2}\right)^{2}$. The dashed, dotted and dash-dotted lines are for $G_{E}^{p}, G_{M}^{p} / \mu_{p}$ and $G_{M}^{n} / \mu_{n}$, respectively. The dotted line and solid line coincide. The dashed line started from 0 is for $G_{E}^{n}$. The experimental data of neutron charge form factor are from Refs. [51-53]. get the best description of the nucleon form factors up to relatively large $Q^{2}$. By comparing with the experimental electromagnetic form factors of nucleon, the best $\Lambda$ is found to be around $0.9 \mathrm{GeV}$. The other two parameters $c_{1}$ and $c_{2}$ are determined by the experimental magnetic moments of proton and neutron. With $\mu_{p}=2.79$ and $\mu_{n}=-1.91$, we get $c_{1}=2.081$ and $c_{2}=0.788$.

Before present the results for strange form factors, we first show the electromagnetic form factors. In Fig. 2, the charge and normalized magnetic form factors of proton and neutron with $\Lambda=0.9 \mathrm{GeV}$ are plotted. The solid line is for the empirical function $1 /\left(1+Q^{2} / 0.71 \mathrm{GeV}^{2}\right)^{2}$. The dashed, dotted, and dash-dotted lines are for $G_{E}^{p}, G_{M}^{p} / \mu_{p}$ and $G_{M}^{n} / \mu_{n}$, respectively. The dotted line is invisible because it coincides with the empirical line. The dashed line started from 0 is for $G_{E}^{n}$. The experimental data of neutron charge form factor are from Refs. [51-53]. From the figure, we can see that our calculated form factors are very close to the experimental data which is a great improvement compared with the results of the traditional chiral effective field theory [22-24].

Now we show the results for the strange form factors. The strange magnetic form factor $G_{M}^{s}\left(Q^{2}\right)$ of nucleon versus $Q^{2}$ with different $\Lambda$ is plotted in Fig. 3. The three solid lines from bottom to top, are for the results with $\Lambda=1,0.9$ and $0.8 \mathrm{GeV}$, respectively. The data with error

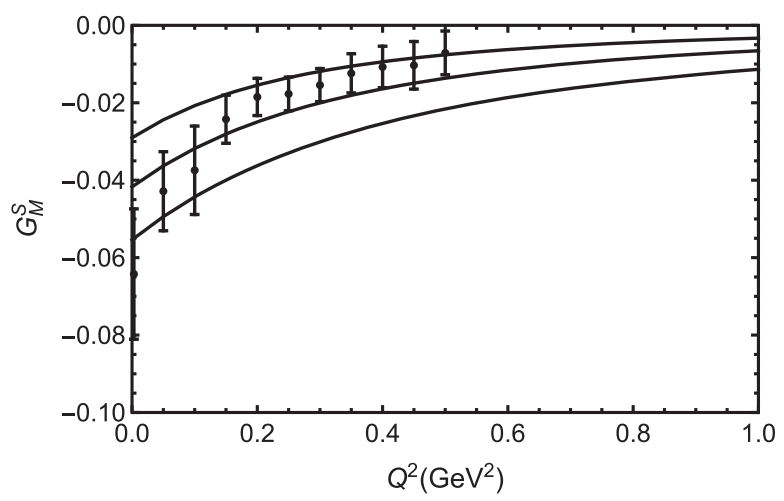

FIG. 3. The strange magnetic form factor of nucleon versus momentum transfer $Q^{2}$ with different $\Lambda$. The three solid lines from bottom to top, are for the results with $\Lambda=1,0.9,0.8 \mathrm{GeV}$, respectively. The data with error bars are from lattice simulation [54]. 
bars from recent lattice simulation [54] are also shown in the figure. The strange magnetic form factors increases with the increasing momentum transfer $Q^{2}$. At zero momentum transfer, when $\Lambda=0.9 \pm 0.1 \mathrm{GeV}, G_{M}^{s}(0)=$ $-0.041_{-0.014}^{+0.012}$. The absolute value of strange magnetic moment in this relativistic chiral Lagrangian is smaller than that in heavy baryon approach with FRR, where $G_{M}^{s}(0)=-0.058_{-0.053}^{+0.034}$ [35]. The main reason for the difference is that rainbow diagrams [Figs. 1(a) and 1(c)] have much smaller contribution to $G_{M}^{s}$ than that in the heavy baryon limit due to the covariant regulator. Though the Kroll-Ruderman and additional diagrams have sizeable contribution to $G_{M}^{s}$ in this relativistic case, while in the heavy baryon limit such contribution is zero, the total absolute value of $G_{M}^{s}(0)$ is a little smaller in relativistic case. Our result is quite different from that in Ref. [55], where heavy baryon ChPT with dimensional regularization was applied. They got a large positive central value of the strange magnetic moment, $\mu_{N}^{s, c v}(0)=0.18$.

The strange charge form factor $G_{E}^{s}\left(Q^{2}\right)$ is plotted in Fig. 4. The three solid lines from top to bottom, are for the results with $\Lambda=1,0.9$, and $0.8 \mathrm{GeV}$, respectively. When $Q^{2}=0, G_{E}^{s}(0)=0$. This is true only when the additional diagrams generated from the expansion of the gauge link are included. The strange charge form factor first increases and then decreases with the increasing $Q^{2}$. At finite $Q^{2}$, $G_{E}^{s}\left(Q^{2}\right)$ is always a small positive number. It is clear that for both strange charge and magnetic form factors, our result is comparable with the lattice data. With the strange form factors, the strange radii can be obtained as

$$
\begin{aligned}
& \left\langle\left(r_{E}^{s}\right)^{2}\right\rangle=-\left.6 \frac{d G_{E}^{s}\left(Q^{2}\right)}{d Q^{2}}\right|_{Q^{2}=0}, \\
& \left\langle\left(r_{M}^{s}\right)^{2}\right\rangle=-\left.6 \frac{d G_{M}^{s}\left(Q^{2}\right)}{d Q^{2}}\right|_{Q^{2}=0} .
\end{aligned}
$$

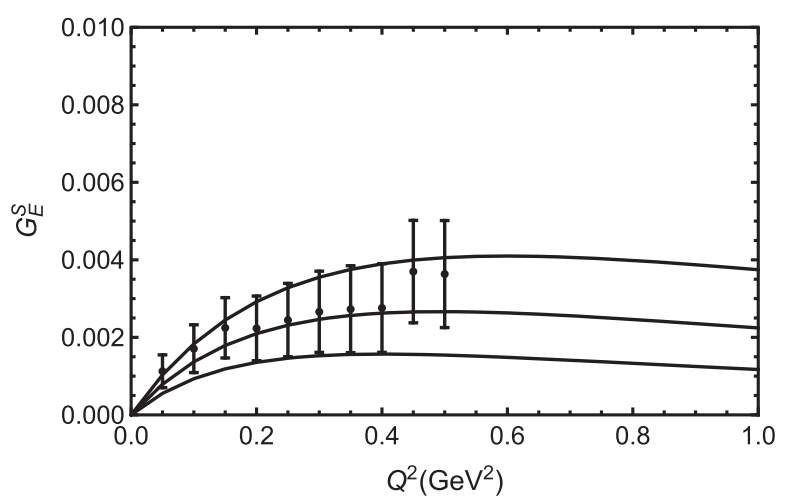

FIG. 4. The strange electric form factor of nucleon versus momentum transfer $Q^{2}$ with different $\Lambda$. The three solid lines from top to bottom, are for the results with $\Lambda=1,0.9$, $0.8 \mathrm{GeV}$, respectively. The data with error bars are from lattice simulation [54].

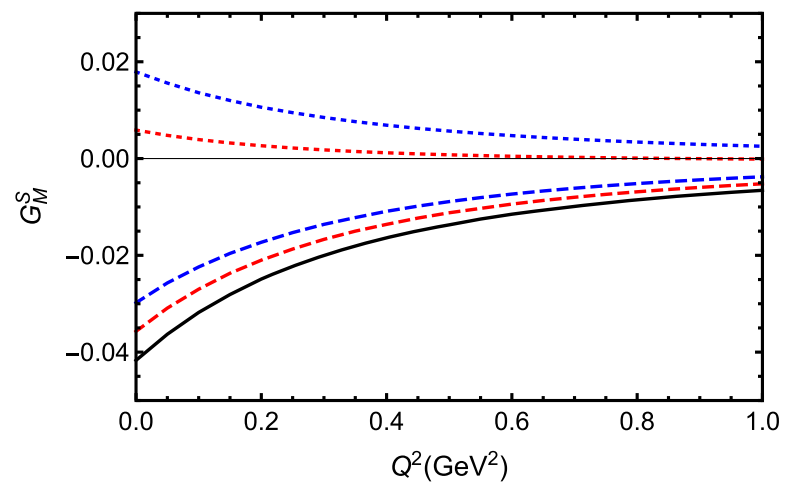

FIG. 5. The proton strange magnetic form factor versus momentum transfer $Q^{2}$ with $\Lambda=0.9 \mathrm{GeV}$. The solid, dashed, and dotted lines are for total, octet, and decuplet contribution to $G_{M}^{s}\left(Q^{2}\right)$, respectively. The red lines are for the normal diagrams and the blue lines are for the additional diagrams.

With $\Lambda=0.9 \pm 0.1 \mathrm{GeV}$, we have $\left\langle\left(r_{E}^{s}\right)^{2}\right\rangle=-0.004 \pm$ $0.001 \mathrm{fm}^{2}$ and $\left\langle\left(r_{M}^{s}\right)^{2}\right\rangle=-0.028 \pm 0.003 \mathrm{fm}^{2}$. Again, our obtained radii are also different from those in traditional heavy baryon ChPT [55], where the electric and magnetic strange radii are $\left\langle\left(r_{E}^{s}\right)^{2}\right\rangle=0.05 \pm 0.09 \mathrm{fm}^{2}$ and $\left\langle\left(r_{M}^{s}\right)^{2}\right\rangle=-0.14 \mathrm{fm}^{2}$.

To see clearly the separate contribution from the octet and decuplet parts, and from the normal diagrams and additional diagrams, in Fig. 5, we plot each contribution to the strange magnetic form factor at $\Lambda=0.9 \mathrm{GeV}$ separately. The solid, dashed, and dotted lines are for total, octet, and decuplet contribution to $G_{M}^{s}\left(Q^{2}\right)$, respectively. The red lines are for the contribution from normal diagrams and the blue lines are for the contribution from additional diagrams. From the figure, one can see that, the octet contribution is dominant. Compared with the octet contribution, the decuplet part gives a smaller opposite number to $G_{M}^{s}$. The additional diagrams also provide important contributions to the total $G_{M}^{s}$.

In Fig. 6, we plot the same curves, but for the strange charge form factor. At $Q^{2}=0$, the contributions from the

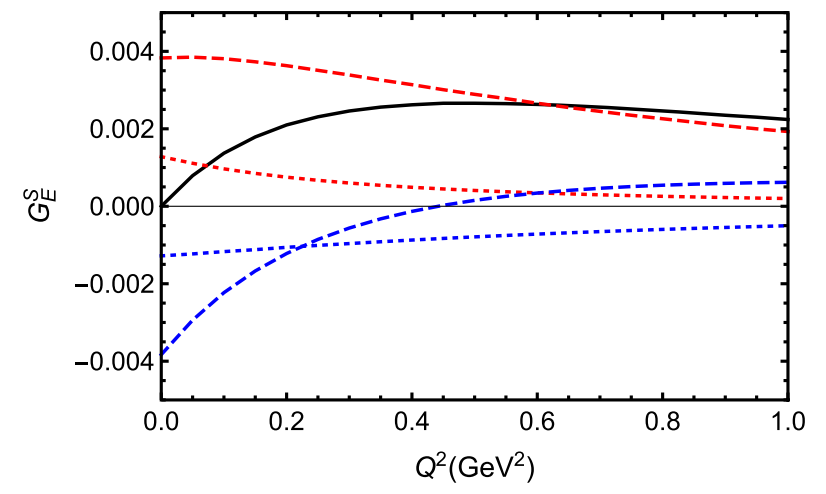

FIG. 6. Same as Fig. 5, but for strange charge form factor $G_{M}^{s}\left(Q^{2}\right)$. 
TABLE I. Contributions to the strange magnetic moment from each diagram and total $G_{M}^{s}(0)$ in unit of $\mu_{N}$. The results from the heavy baryon formalism [35] are also listed in the last line.

\begin{tabular}{lcccccccccccc}
\hline \hline$\Lambda(\mathrm{GeV})$ & $1 a$ & $1 b$ & $1 c$ & $1 d+1 e$ & $1 f+1 g$ & $1 h$ & $1 i$ & $1 j$ & $1 k+1 l$ & $1 m+1 n$ & $1 o+1 p$ & $G_{M}^{s}(0)$ \\
\hline 0.8 & -0.011 & 0.003 & -0.005 & -0.010 & -0.021 & 0.005 & -0.0002 & 0.005 & -0.007 & 0.0007 & 0.011 & -0.029 \\
0.9 & -0.017 & 0.005 & -0.008 & -0.016 & -0.030 & 0.009 & -0.0004 & 0.009 & -0.012 & 0.001 & 0.018 & -0.041 \\
1 & -0.024 & 0.009 & -0.011 & -0.026 & -0.039 & 0.013 & -0.0008 & 0.014 & -0.018 & 0.002 & 0.026 & -0.055 \\
0.8 & -0.050 & $\cdots$ & -0.021 & $\cdots$ & $\cdots$ & 0.009 & $\cdots$ & 0.013 & -0.009 & $\cdots$ & $\cdots$ & -0.058 \\
\hline \hline
\end{tabular}

normal and additional diagrams cancel each other. As a result, the net strangeness is zero. This is guaranteed by the gauge symmetry of strange quark. Similar as in the magnetic case, the octet contribution is dominant for the total $G_{E}^{s}\left(Q^{2}\right)$. At small $Q^{2}$, the contribution from the additional diagrams changes more quickly than that from the normal diagrams. Therefore, $G_{E}^{s}$ first increases from 0 and then decreases smoothly with the increasing $Q^{2}$.

\section{SUMMARY}

We studied the strange form factors of nucleon with the nonlocal chiral effective Lagrangian. Both the octet and decuplet intermediate states are included in the one loop calculation. The covariant form factors are derived from the nonlocal Lagrangian. This is the relativistic version of the finite-range-regularization, which make it possible to study the hadron structure at relatively large $Q^{2}$. From the previous study of the nucleon electromagnetic form factors, it shows this nonlocal Lagrangian method is a great improvement compared with the traditional chiral effective theory. The gauge link is introduced to guarantee the local gauge symmetry. As a result, in addition to the normal diagrams which are generated from the minimal substitution, the additional diagrams appear which are generated from the expansion of the gauge link. These additional diagrams are crucial to get the net strangeness zero at $Q^{2}=$ 0 for nucleon. They also have important contribution to the magnetic form factors. For both $G_{E}^{s}$ and $G_{M}^{s}$, the octet intermediated states provide more important contribution than decuplet intermediate states. In this nonlocal chiral effective Lagrangian, there are three free parameters. $c_{1}$ and $c_{2}$ are determined by the experimental magnetic moments of proton and neutron. $\Lambda$ in the correlation function is determined by the best description of the nucleon electromagnetic form factors up to relatively large $Q^{2}$. At finite momentum transfer, the strange charge form factor is positive, while the strange magnetic form factor is negative. At $Q^{2}=0$, the strange magnetic moment is $-0.041_{-0.014}^{+0.012}$. Compared with the calculated $G_{M}^{s}(0)$ in heavy baryon formalism with finite-range-regularization, the absolute value of $G_{M}^{s}(0)$ calculated in this relativistic version is a little smaller. Our results are also comparable with the recent lattice simulation. As a summary, we list the contribution to the strange magnetic moment of each diagram in Table I.

\section{ACKNOWLEDGMENTS}

This work is supported by the National Natural Sciences Foundations of China under the Grant No. 11475186, the Sino-German CRC 110 "Symmetries and the Emergence of Structure in QCD" project by NSFC under the Grant No. 11621131001, and the Key Research Program of Frontier Sciences, CAS under Grant No. Y7292610K1.

\section{APPENDIX: DECUPLET CONTRIBUTION}

The expressions for the decuplet part are written in the following way. The contribution of Fig. 1(h) is expressed as

$$
\Gamma_{h}^{\mu(p)}=\frac{\mathcal{C}^{2}}{4 f^{2}} I_{h K}^{N \Sigma^{*}},
$$

where

$$
\begin{aligned}
I_{h K}^{N \Sigma^{*}}= & \left.\bar{u}\left(p^{\prime}\right) \tilde{F}(q) \int \frac{d^{4} k}{(2 \pi)^{4}}\left((k+q)_{\sigma}+z(\not k+\not q) \gamma_{\sigma}\right)\right) \tilde{F}(q+k) \frac{1}{D_{K}(k+q)}(2 k+q)^{\mu} \\
& \left.\times \frac{1}{D_{K}(k)} \frac{1}{\not p-\not k-m_{\Sigma^{*}}} S_{\sigma \rho}(p-k)\left(-k_{\rho}-z \gamma_{\rho} \not k\right)\right) \tilde{F}(k) u(p) .
\end{aligned}
$$

$S_{\sigma \rho}$ is expressed as

$$
S_{\sigma \rho}(k)=-g_{\sigma \rho}+\frac{\gamma_{\sigma} \gamma_{\rho}}{3}+\frac{2 k_{\sigma} k_{\rho}}{3 m_{\Sigma^{*}}^{2}}+\frac{\gamma_{\sigma} k_{\rho}-\gamma_{\rho} k_{\sigma}}{3 m_{\Sigma^{*}}}
$$


The contribution of Fig. 1(i) is expressed as

$$
\Gamma_{i}^{\mu(p)}=\frac{\mathcal{C}^{2}}{4 f^{2}} \frac{4 m_{\Sigma^{*}}^{2}+c_{1} Q^{2}}{4 m_{\Sigma^{*}}^{2}+Q^{2}} I_{i K}^{N \Sigma^{*}}
$$

where

$$
\begin{aligned}
I_{i K}^{N \Sigma^{*}}= & \bar{u}\left(p^{\prime}\right) \tilde{F}(q) \int \frac{d^{4} k}{(2 \pi)^{4}}\left(k_{\sigma}+z \not k \gamma_{\sigma}\right) \tilde{F}(k) \frac{1}{D_{K}(k)} \frac{1}{\not p^{\prime}-\not k-m_{\Sigma^{*}}} S_{\sigma \alpha}\left(p^{\prime}-k\right) \\
& \times\left(\gamma^{\alpha \beta \mu}\right) \frac{1}{\not p-\not k-m_{\Sigma^{*}}} S_{\beta \rho}\left(k_{\rho}+z \gamma_{\rho} \not k\right) F(k) u(p) .
\end{aligned}
$$

The contribution of Fig. 1(j) is expressed as

$$
\Gamma_{j}^{\mu(p)}=\frac{\mathcal{C}^{2}}{4 f^{2}} \frac{4 m_{\Sigma^{*}}^{2}}{4 m_{\Sigma^{*}}^{2}+Q^{2}} I_{j K}^{N \Sigma^{*}},
$$

where

$$
\begin{aligned}
I_{j K}^{N \Sigma^{*}}= & \bar{u}\left(p^{\prime}\right) \tilde{F}(q) \int \frac{d^{4} k}{(2 \pi)^{4}}\left(k_{\sigma}+z \not k \gamma_{\sigma}\right) \tilde{F}(k) \frac{1}{D_{K}(k)} \frac{1}{\not p^{\prime}-\not k-m_{\Sigma^{*}}} S_{\sigma \nu}\left(p^{\prime}-k\right) \frac{\left(1-c_{1}\right)}{2 m_{\Sigma^{*}}} \sigma^{\mu \lambda} q_{\lambda} \\
& \times \frac{i}{\not p-\not k-m_{\Sigma^{*}}} S_{\nu \rho}(p-k)\left(k_{\rho}+z \gamma_{\rho} \not k\right) \tilde{F}(k) u(p) .
\end{aligned}
$$

The contribution of Figs. 1(k) and 1(1) is expressed as

$$
\Gamma_{k+l}^{\mu(p)}=\frac{\mathcal{C}(D-F) c_{1}}{4 m_{\Sigma^{*}} f^{2}} I_{(k+l) K}^{\Sigma \Sigma^{*}},
$$

where

$$
\begin{aligned}
I_{(k+l) K}^{\Sigma \Sigma^{*}}= & \bar{u}\left(p^{\prime}\right) \tilde{F}(q) \int \frac{d^{4} k}{(2 \pi)^{4}} \tilde{F}^{2}(k) \not k \gamma_{5} \frac{1}{\not p^{\prime}-\not k-m_{\Sigma^{*}}} \not \gamma_{5} \frac{1}{\not p-\not k-m_{\Sigma^{*}}} S_{\mu \rho}(p-k)\left(k_{\rho}+z \gamma_{\rho} \not k\right) \frac{1}{D_{K}(k)} u(p) \\
& -\bar{u}\left(p^{\prime}\right) \tilde{F}(q) \int \frac{d^{4} k}{(2 \pi)^{4}} \tilde{F}^{2}(k) \not k \gamma_{5} \frac{1}{\not p^{\prime}-\not k-m_{\Sigma^{*}}} \gamma^{\mu} \gamma_{5} q_{\nu} \frac{1}{\not p-\not k-m_{\Sigma^{*}}} S_{\nu \rho}(p-k)\left(k_{\rho}+z \gamma_{\rho} \not k\right) \frac{1}{D_{K}(k)} u(p) \\
& +\bar{u}\left(p^{\prime}\right) \tilde{F}(q) \int \frac{d^{4} k}{(2 \pi)^{4}} \tilde{F}^{2}(k)\left(k_{\nu}+z \not k \gamma_{\nu}\right) \frac{1}{\not p^{\prime}-\not k-m_{\Sigma^{*}}} S_{\nu \rho}\left(p^{\prime}-k\right) q_{\rho} \gamma^{\mu} \gamma_{5} \frac{1}{\not p-\not k-m_{\Sigma^{*}}} \not k \gamma_{5} \frac{1}{D_{K}(k)} u(p) \\
& -\bar{u}\left(p^{\prime}\right) \tilde{F}(q) \int \frac{d^{4} k}{(2 \pi)^{4}} \tilde{F}^{2}(k)\left(k_{\nu}+z \not k \gamma_{\nu}\right) \frac{1}{\not p^{\prime}-\not k-m_{\Sigma^{*}}} S_{\nu \mu}\left(p^{\prime}-k\right) q_{\rho} \gamma^{\rho} \gamma_{5} \frac{1}{\not p-\not k-m_{\Sigma^{*}}} \not k \gamma_{5} \frac{1}{D_{K}(k)} u(p) .
\end{aligned}
$$

The contribution of Figs. 1(m) and 1(n) is expressed as

$$
\Gamma_{m+n}^{\mu(p)}=\frac{\mathcal{C}^{2}}{8 f^{2}} I_{(m+n) K}^{N \Sigma^{*}},
$$

where

$$
\begin{aligned}
I_{(m+n) K}^{N \Sigma^{*}}= & \bar{u}\left(p^{\prime}\right) \tilde{F}(q) \int \frac{d^{4} k}{(2 \pi)^{4}}\left(k_{\sigma}+z \not k \gamma_{\sigma}\right) \tilde{F}^{2}(k) \frac{1}{D_{K}(k)} \frac{1}{\not p^{\prime}-\not k-m_{\Sigma^{*}}} S_{\sigma \rho}\left(p^{\prime}-k\right)\left(g^{\rho \mu}+z \gamma^{\rho} \gamma^{\mu}\right) u(p) \\
& +\bar{u}\left(p^{\prime}\right) \tilde{F}(q) \int \frac{d^{4} k}{(2 \pi)^{4}}\left(g^{\sigma \mu}+z \gamma^{\mu} \gamma^{\sigma}\right) \tilde{F}^{2}(k) \frac{1}{D_{K}(k)} \frac{1}{\not p-\not k-m_{\Sigma^{*}}} S_{\sigma \rho}(p-k)\left(k_{\rho}+z \gamma_{\rho} \not k\right) u(p) .
\end{aligned}
$$


The contribution of Figs. 1(o) and 1(p) is expressed as

$$
\Gamma_{o+p}^{\mu(p)}=\frac{\mathcal{C}^{2}}{8 f^{2}} I_{(o+p) K}^{N \Sigma^{*}}
$$

where

$$
\begin{aligned}
I_{(o+p) K}^{N \Sigma^{*}}= & -\bar{u}\left(p^{\prime}\right) \tilde{F}(q) \int \frac{d^{4} k}{(2 \pi)^{4}}\left(k_{\sigma}+z \not k \gamma_{\sigma}\right) \tilde{F}(k) \frac{1}{D_{K}(k)} \frac{1}{\not p^{\prime}-\not k-m_{\Sigma^{*}}} S_{\sigma \rho}\left(p^{\prime}-k\right)\left(k_{\rho}-q_{\rho}+z \gamma_{\rho}(\not k-\not q)\right) \\
& \times \frac{(-2 k+q)^{\mu}}{-2 k q+q^{2}}[F(k-q)-F(k)] u(p) \\
& +\bar{u}\left(p^{\prime}\right) \tilde{F}(q) \int \frac{d^{4} k}{(2 \pi)^{4}}\left(k_{\sigma}+q_{\sigma}+z(\not k+\not q) \gamma_{\sigma}\right) \frac{(2 k+q)^{\mu}}{2 k q+q^{2}}[F(k+q)-F(k)] \frac{1}{D_{K}(k)} \\
& \times \frac{1}{\not p-\not k-m_{\Sigma^{*}}} S_{\sigma \rho}(p-k) \tilde{F}(k)\left(k_{\rho}+z \gamma_{\rho} \not k\right) u(p) .
\end{aligned}
$$

[1] D. T. Spayde et al. (SAMPLE Collaboration), Phys. Lett. B 583, 79 (2004).

[2] F. E. Maas et al. (A4 Collaboration), Phys. Rev. Lett. 93, 022002 (2004).

[3] F. E. Maas et al., Phys. Rev. Lett. 94, 152001 (2005).

[4] D. S. Armstrong et al. (G0 Collaboration), Phys. Rev. Lett. 95, 092001 (2005).

[5] A. Acha et al. (HAPPEX Collaboration), Phys. Rev. Lett. 98, 032301 (2007).

[6] K. A. Aniol et al. (HAPPEX Collaboration), Phys. Lett. B 635, 275 (2006).

[7] K. A. Aniol et al. (HAPPEX Collaboration), Phys. Rev. C 69, 065501 (2004).

[8] R. D. Young, J. Roche, R. D. Carlini, and A. W. Thomas, Phys. Rev. Lett. 97, 102002 (2006).

[9] R. González-Jiménez, J. A. Caballero, and T. W. Donnelly, Phys. Rev. D 90, 033002 (2014).

[10] D.-H. Lu, A. W. Thomas, and A. G. Williams, Phys. Rev. C 57, 2628 (1998).

[11] K. Berger, R. F. Wagenbrunn, and W. Plessas, Phys. Rev. D 70, 094027 (2004).

[12] B. Julia-Diaz, D. O. Riska, and F. Coester, Phys. Rev. C 69 , 035212 (2004); 75, 069902(E) (2007).

[13] A. J. Buchmann and R. F. Lebed, Phys. Rev. D 67, 016002 (2003).

[14] S. Cheedket, V. E. Lyubovitskij, T. Gutsche, A. Faessler, K. Pumsa-ard, and Y. Yan, Eur. Phys. J. A 20, 317 (2004).

[15] V. E. Lyubovitskij, P. Wang, T. Gutsche, and A. Faessler, Phys. Rev. C 66, 055204 (2002).

[16] R. A. Williams and C. Puckett-Truman, Phys. Rev. C 53, 1580 (1996).

[17] P.-N. Shen, Y.-B. Dong, Z.-Y. Zhang, Y.-W. Yu, and T. S. H. Lee, Phys. Rev. C 55, 2024 (1997).

[18] R. Jakob, P. Kroll, M. Schurmann, and W. Schweiger, Z. Phys. A 347, 109 (1993).
[19] G. Hellstern and C. Weiss, Phys. Lett. B 351, 64 (1995).

[20] J. Gegelia and G. Japaridze, Phys. Rev. D 60, 114038 (1999).

[21] T. Fuchs, J. Gegelia, G. Japaridze, and S. Scherer, Phys. Rev. D 68, 056005 (2003).

[22] T. Fuchs, J. Gegelia, and S. Scherer, J. Phys. G 30, 1407 (2004).

[23] B. Kubis and U.-G. Meissner, Nucl. Phys. A679, 698 (2001).

[24] T. Bauer, J. C. Bernauer, and S. Scherer, Phys. Rev. C 86, 065206 (2012).

[25] R. D. Young, D. B. Leinweber, and A. W. Thomas, Prog. Part. Nucl. Phys. 50, 399 (2003).

[26] D. B. Leinweber, A. W. Thomas, and R. D. Young, Phys. Rev. Lett. 92, 242002 (2004).

[27] P. Wang, D. B. Leinweber, A. W. Thomas, and R. D. Young, Phys. Rev. D 75, 073012 (2007).

[28] P. Wang and A. W. Thomas, Phys. Rev. D 81, 114015 (2010).

[29] C. R. Allton, W. Armour, D. B. Leinweber, A. W. Thomas, and R. D. Young, Phys. Lett. B 628, 125 (2005).

[30] W. Armour, C. R. Allton, D. B. Leinweber, A. W. Thomas, and R. D. Young, Nucl. Phys. A840, 97 (2010).

[31] J. M. M. Hall, D. B. Leinweber, and R. D. Young, Phys. Rev. D 88, 014504 (2013).

[32] D. B. Leinweber, S. Boinepalli, I. C. Cloet, A. W. Thomas, A. G. Williams, R. D. Young, J. M. Zanotti, and J. B. Zhang, Phys. Rev. Lett. 94, 212001 (2005).

[33] P. Wang, D. B. Leinweber, A. W. Thomas, and R. D. Young, Phys. Rev. C 79, 065202 (2009).

[34] P. Wang, D. B. Leinweber, A. W. Thomas, and R. D. Young, Phys. Rev. D 86, 094038 (2012).

[35] P. Wang, D. B. Leinweber, and A. W. Thomas, Phys. Rev. D 89, 033008 (2014).

[36] J. M. M. Hall, D. B. Leinweber, and R. D. Young, Phys. Rev. D 89, 054511 (2014).

[37] P. Wang, D. B. Leinweber, and A. W. Thomas, Phys. Rev. D 92, 034508 (2015). 
[38] H. Li, P. Wang, D. B. Leinweber, and A. W. Thomas, Phys. Rev. C 93, 045203 (2016).

[39] H. Li and P. Wang, Chin. Phys. C 40, 123106 (2016).

[40] P. Wang, D. B. Leinweber, A. W. Thomas, and R. D. Young, Phys. Rev. D 79, 094001 (2009).

[41] P. Wang, Can. J. Phys. 92, 25 (2014).

[42] F. He and P. Wang, Phys. Rev. D 97, 036007 (2018).

[43] E. E. Jenkins, Nucl. Phys. B368, 190 (1992).

[44] E. E. Jenkins, M. E. Luke, A. V. Manohar, and M. J. Savage, Phys. Lett. B 302, 482 (1993); 388, 866(E) (1996).

[45] S. Scherer, Adv. Nucl. Phys. 27, 277 (2003).

[46] P. Ha and L. Durand, Phys. Rev. D 67, 073017 (2003).

[47] P. Ha, Phys. Rev. D 58, 113003 (1998).
[48] J. Terning, Phys. Rev. D 44, 887 (1991).

[49] V. Pascalutsa, M. Vanderhaeghen, and S. N. Yang, Phys. Rep. 437, 125 (2007).

[50] L. M. Nath, B. Etemadi, and J. D. Kimel, Phys. Rev. D 3, 2153 (1971).

[51] M. Seimetz (A1 Collaboration), Nucl. Phys. A755, 253 (2005).

[52] A. Semenov, AIP Conf. Proc. 1257, 661 (2010).

[53] V. Sulkosky et al., Phys. Rev. C 96, 065206 (2017).

[54] R. S. Sufian, Y.-B. Yang, J. Liang, T. Draper, and K.-F. Liu, Phys. Rev. D 96, 114504 (2017).

[55] T. R. Hemmert, B. Kubis, and U.-G. Meissner, Phys. Rev. C 60, 045501 (1999). 